\title{
Icariin recovers the osteogenic differentiation and bone formation of bone marrow stromal cells from a rat model of estrogen deficiency-induced osteoporosis
}

\author{
ZHIQIANG LUO ${ }^{1 *}$, MINGLU LIU ${ }^{1,2^{*}}$, LIKUN SUN $^{3}$ and FEILONG RUI ${ }^{4}$ \\ ${ }^{1}$ The Second Clinical Hospital of Lanzhou University, Lanzhou, Gansu 730000; ${ }^{2}$ The Second Out-Patient Department, \\ National Defence University of PLA China, Beijing 100039; ${ }^{3}$ School of Life Sciences, Lanzhou University, Lanzhou, \\ Gansu 730000; ${ }^{4}$ School of Physical Education, Tianshui Normal University, Tianshui, Gansu 741000, P.R. China
}

Received February 2, 2014; Accepted November 7, 2014

DOI: $10.3892 / \mathrm{mmr} .2015 .3369$

\begin{abstract}
A number of recent studies have suggested that icariin (ICA), a class of phytochemical with numerous biological activities, may exert protective effects against postmenopausal bone loss. However, it remains unclear whether ICA regulates or improves the osteoblastic function of bone marrow stromal cells (BMSCs) in the treatment and prevention of osteoporosis. In the present study, the osteogenic differentiation of BMSCs from ovariectomy (OVX) rats was found to be significantly decreased in vitro compared with that in rats that had undergone a sham operation. Treatment with ICA at a dose of 10-5 M was shown to restore the osteogenic differentiation of BMSCs in OVX rats. The results indicated that ICA restored the differentiation and mineralization capacity of OVX-BMSCs, which had been induced by estrogen deficiency. The effects of this compound on alkaline phosphatase (ALP) activity and calcium deposition were also measured at various time points. The number of colonies and areas that stained positive for ALP expression, and mineralized bone nodules were analyzed histochemically at 14 and 21 days after the osteogenic induction. The expression of the runt-related transcription factor 2 and osterix bone metabolism biomarker proteins and genes were detected by western blotting and reverse transcription-quantitative polymerase chain reaction (RT-qPCR). The expression of factors involved in the estrogen signaling pathway, estrogen receptor $\alpha(\mathrm{ER} \alpha)$, progesterone receptor (PR) and trefoil factor 1 (PS-2), was also detected by western blotting and RT-qPCR. ICA enhanced the expression of ER $\alpha$, PR, PS-2 in OVX-BMSCs, but this effect
\end{abstract}

Correspondence to: Professor Minglu Liu, The Second Out-Patient Department, National Defence University of PLA China, 83 Fuxing Road, Haidian, Beijing 100039, P.R. China

E-mail:1ml.2007@163.com

*Contributed equally

Key words: mesenchymal stem cells, icariin, osteoporosis, differentiation, mineralization was abrogated when ICI 182780, an ER antagonist was added. Transplantation of BMSCs into nude mice demonstrated that ICA restored the osteogenic capability of OVX-BMSCs in vivo. Therefore, it may be that ICA acts through the estrogen pathway in order to improve and restore the osteogenic differentiation and mineralization of OVX-BMSCs, which are inhibited by estrogen deficiency and increasing age.

\section{Introduction}

Osteoporosis is a clinically significant problem worldwide, the incidence of which increases with age. It is a skeletal disorder characterized by impaired bone strength, which predisposes to fractures without an identifiable injury or with minimal trauma, which would be insufficient to fracture healthy bone $(1,2)$. Therefore, bone regeneration is also a significant problem in osteoporosis. Bone mesenchymal stem cells (BMSCs) are important in bone tissue repair and regeneration. For example, these cells are known to progressively differentiate into pre-osteoblasts and mature osteoblasts under appropriate stimulation from regulatory factors present in the microenvironment $(3,4)$. Shi et al $(5)$ reported that venous injection of BMSCs into mice that had undergone ovariectomy (OVX), prevented or reversed osteoporosis. These results suggested that BMSC-based treatment of osteoporosis may be a promising choice, regardless on the location of bone disease, or whether it is due to a systemic cause. Postmenopausal osteoporosis is one of the clinical manifestations of estrogen deficiency, which occurs during the process of bone aging. Osteoporotic bone loss is the result of high bone turnover, in which bone resorption outpaces the rate of bone deposition $(6,7)$. This imbalance in bone turnover is induced by estrogen deficiency in women and female rodents and may be ameliorated by treatment with bioavailable estrogens, including selective estrogen receptor modulators (SERMs) (8). Estrogen and SERMs primarily act by regulating gene transcription via estrogen receptors (ERs; $\mathrm{ER} \alpha$ and $\mathrm{ER} \beta)(9,10)$. ERs belong to the nuclear receptor gene superfamily and have been shown to act as ligand-inducible transcriptional factors (11). ER dimers directly or indirectly associate with specific DNA elements in the target gene promoter and control transcription by reorganizing chromatin 
structure and histone modifications $(10,12)$. The present study focused on the association between postmenopausal osteoporosis and the osteogenic differentiation of BMSCs or ER changes (13). These changes may be modulated by certain small molecular compounds of estradiol and flavonoids.

Recently, Chen et al $(14,15)$ demonstrated that icariin (ICA) enhances osteogenic differentiation of rat BMSCs, improves the maturation and mineralization of osteoblasts in vitro, and suppresses osteoclastogenesis and inhibits bone resorption activity in vivo (16-20). $10^{-5} \mathrm{M}$ ICA has been shown to be a potent stimulator of osteogenic differentiation in vitro (19-21). It has been demonstrated that it stimulates the proliferation of rat BMSCs and increases the number of colony-forming units of fibroblasts that stain positive for alkaline phosphatase (ALP). In addition, it promotes ALP activity, osteocalcin secretion and calcium deposition in rat BMSCs in a dose-dependent manner, suggesting a potential anabolic effect on bone (19-22). Mok et al (23) reported that ICA exerts an anabolic effect in the bone possibly via the activation of ER in a ligand-independent manner, and as a result of its ability to prevent OVX-induced bone loss. However, the mechanisms underlying the osteogenic differentiation of BMSCs, in response to treatment with ICA, in an OVX-induced model of osteoporosis remain unclear. The current study focused on the effect of $10^{-5} \mathrm{M}$ ICA on BMSCs from an OVX-induced rat model of osteoporosis, as well as the possible underlying mechanisms of these effects, in vitro and in vivo.

\section{Materials and methods}

Animal models. Twelve female Wistar rats (eight weeks old) weighing 80-100 $\mathrm{g}$ were obtained from the Animal Breeding Center of Gansu College of Traditional Chinese Medicine (Lanzhou, China). All experimental animals were housed under standard conditions $\left(22^{\circ} \mathrm{C}, 12 \mathrm{~h}\right.$ light $/ 12 \mathrm{~h}$ dark cycles and $50-55 \%$ humidity), and the experimental protocol was approved by the Animal Ethical Committee of Lanzhou University (Lanzhou, China). All rats were randomly divided into two group, which were subjected to either OVX or a sham operation (Sham). At three months post-surgery, three rats from each group were sacrificed via intraperitoneal injection of $1 \%$ pentobarbital. The metaphysis regions of the distal femur and femoral shaft were scanned by micro-computed tomography (Siemens Inveon Micro CT; Siemens, Munich, Germany) with a source voltage of $80 \mathrm{keV}$, current of $500 \mu \mathrm{A}$ and $14.97 \mu \mathrm{m}$ isotropic resolution. This was to ensure the successful establishment of the OVX animal model. Following 3D reconstruction, bone volume fraction and bone mineral density (BMD) were calculated using the built-in software. Procedures for obtaining bone marrow samples (in order to isolating bone marrow stromal cells) from a further six rats were conducted according to the Guide for the Care and Use of Laboratory Animals, published by the US National Institutes of Health.

Reagents. ICA (purity $>98 \%$ ) was purchased from the National Institute for Control of Pharmaceutical and Biological Products (Beijing, China). Dulbecco's modified Eagle's medium with F12 (DMEM/F12) and fetal bovine serum (FBS) were purchased from Invitrogen Life Technologies (Grand Island, NY, USA). Penicillin and streptomycin were obtained from Gibco BRL (Gaithersburg, MD, USA). The majority of drugs used were purchased from Sigma-Aldrich (Steinheim, Germany), including dexamethasone, b-glycerophosphate, ascorbic acid phosphate and 1-naphthyl phosphate sodium salt monohydrate. The alkaline phosphatase activity measurement kit was purchased from Nanjing Jiancheng Company (Nanjing, China) and the calcium colorimetric assay kit was obtained from Biovision (San Francisco, CA, USA). All other chemicals were of analytical grade.

Isolation and culture of sham and OVX rat BMSCs. Following euthanasia, BMSCs were harvested from the tibial and femoral bone marrow of Sham and OVX rats, and cultured in low glucose DMEM, low glucose; (Gibco-BRL, Grand Island, NY, USA) and supplemented with $10 \%$ fetal bovine serum (Hyclone, Logan, UT, USA), containing $100 \mathrm{U} / \mathrm{ml}$ penicillin, $100 \mathrm{U} / \mathrm{ml}$ streptomycin and $2 \mathrm{mM}$ L-glutamine (Sigma-Aldrich) as previously described (22). BMSCs were incubated for $24 \mathrm{~h}$ at $37^{\circ} \mathrm{C}$ in a humidified atmosphere of $95 \%$ air and $5 \% \mathrm{CO}_{2}$, following which the medium was changed in order to discard non-adherent cells. The medium was subsequently refreshed every three days. When confluence reached $\sim 80 \%$, cells were passaged for expansion. Cells were at passage three were used for the subsequent experiments.

\section{Comparison of proliferation and differentiation between} Sham-BMSC and OVX-BMSC groups. The proliferation of BMSCs from each group was determined by an MTT assay (Sigma-Aldrich). Briefly, cells were seeded at a density of $1 \times 10^{3}$ cells/well on a 96 -well plate in triplicate in the DMEM medium containing $1 \%$ fetal bovine serum for, 1, 3, 5 and 7 days. MTT $(5 \mathrm{mg} / \mathrm{ml})$ was added to each well and incubated for $4 \mathrm{~h}$. The medium was removed and dimethyl sulfoxide (DMSO; Sigma-Aldrich) was then added in order to dissolve formazan. The absorbance value was measured using a microplate reader (BioTek Instruments, Winooski, VT, USA) at $490 \mathrm{~nm}$. The results are expressed as units of optical density absorbance values.

The differentiation of BMSCs in each group was determined by measuring alkaline phosphatase (ALP) activity and ALP staining at day 7 in an osteogenic medium containing $10^{-8} \mathrm{M}$ dexamethasone, $50 \mu \mathrm{g} / \mathrm{ml} \mathrm{L}$-2-ascorbic acid, and $10 \mathrm{~mm}$ $\beta$-glycerophosphate. The ALP activity was measured using a commercial kit as instructed (Nanjing Jiancheng Company). A modified King's method (24) was used in the kit and the results are expressed as nmol of phenol/15 $\mathrm{min} / \mathrm{mg}$ protein. Protein concentrations were determined using a bicinchoninic acid (BCA) protein assay kit (Nanjing Jiancheng Company). The numbers of colonies positive for ALP (CFU-FALP) were also compared in each group on day 12. Cells were fixed in $3.7 \%$ formaldehyde and 90\% ethanol solution (Zhong Shan Jin Qiao Company, Beijing, China) for $5 \mathrm{~min}$, washed and then stained for $15 \sim 20 \mathrm{~min}$ at $37^{\circ} \mathrm{C}$ in $20 \mathrm{ml}$ Michalis buffer, $\mathrm{pH} 8.9$, containing $10 \mathrm{mg}$ 1-naphthyl phosphate sodium and $10 \mathrm{mg}$ fast blue RR salt (Sigma-Aldrich). In order to detect mineralization, alizarin red staining (Zhong Shan Jin Qiao Company) of mineralized nodules was conducted on day 14 . Briefly, the cells were fixed in $3.7 \%$ formaldehyde for $10 \mathrm{~min}$ and stained by $0.1 \%$ alizarin red for $1 \mathrm{~h}$ at $37^{\circ} \mathrm{C}$. The calcium deposition volume was measured using a calcium colorimetric assay kit (BioVision, Inc., Milpitas, CA, USA). 
Runt-related transcription factor 2 (Runx-2) and Osterix (OSX) are two transcription factors required for osteoblast differentiation and bone formation (25). The expression of the Runx-2 and OSX proteins an genes was detected by western blotting and reverse transcription-quantitative polymerase chain reaction (RT-qPCR). Sham-BMSC and OVX-BMSCs were cultured for seven days in an osteogenic medium. The cells were washed twice with distilled water and total protein was collected by adding lysis buffer (Zhongshan Goldenbridge, Beijing, China). Protein concentration was measured using the BCA protein assay kit, according to the manufacturer's instructions (Beyotime Institute of Biotechnology, Shanghai, China). Total protein $(50 \mu \mathrm{g})$ from each sample was separated by SDS-PAGE (12\% gel) and transferred to polyvinylidene fluoride membranes (EMD Millipore, Billerica, MA, USA). Following incubation in blocking solution ( $2 \%$ non-fat milk) for $2 \mathrm{~h}$ at room temperature, membranes were incubated for overnight at $4^{\circ} \mathrm{C}$ with the following primary antibodies at 1:1,000 dilution: Mouse anti-Runx 2 or OSX (Abcam, Hong Kong), and mouse anti $\beta$-actin polyclonal antibody (Zhongshan Goldenbridge). Following three washes with Tris-buffered saline with Tween 20 (Beyotime Institute of Biotechnology), membranes were incubated with 1:3,000 dilution of the secondary antibody (Zhongshan Goldenbridge) for $2 \mathrm{~h}$, and the immunoreactions signals were detected using the enhanced chemiluminescence reagent (EMD Millipore). Total RNA was extracted from BMSCs in the OVX and Sham groups following culture in osteogenic medium for 7 days, using TRIzol $^{\circledR}$ reagent (Invitrogen Life Technologies). RNA $(1 \mu \mathrm{g})$ was reverse transcribed with PrimeScript RT reagent kit (Takara Bio, Inc., Kyoto, Japan) according to the manufacturer's instructions. The cDNA amplification and detection was performed in triplicate with the Bio-Rad iQ5 real-time PCR system (Bio-Rad, Hercules, CA, USA) using a SYBR Premix Ex Taq kit (Takara Bio, Inc.). The relative gene expression level was normalized to that of the reference gene, $\beta$-actin, based on the $2^{-\Delta \Delta \mathrm{Ct}}$ method. Cycling conditions were as follows: Preincubation $\left(95^{\circ} \mathrm{C}\right.$ for $10 \mathrm{~min}), 40$ cycles were the performed, comprising denaturation $\left(94^{\circ} \mathrm{C}\right.$ for $\left.30 \mathrm{sec}\right)$, annealing $\left(60^{\circ} \mathrm{C}\right.$ for $\left.1 \mathrm{~min}\right)$ and extension $\left(72^{\circ} \mathrm{C}\right.$ for $1 \mathrm{~min}$ ). A melting curve was acquired using $95^{\circ} \mathrm{C}$ for $15 \mathrm{sec}, 60^{\circ} \mathrm{C}$ for $30 \mathrm{sec}$ and $95^{\circ} \mathrm{C}$ for $15 \mathrm{sec}$.

ICA treatment the OVX-BMSC to recover the cell osteogenic differentiation assay and the mechanisms analysis. BMSCs from the Sham and OVX groups were planted in 12-well and six-well plates, respectively. In some culture wells, medium was supplemented with ICA at $10^{-5} \mathrm{~mol} / \mathrm{l}$, a concentration that has bee shown to be osteogenic in BMSCs (14). The final concentration of DMSO, used as a solvent of ICA, in the culture was $>0.05 \%$, which did not interfere with the test system (26). In the cell differentiation assay, the OVX-BMSC cells were randomly divided into four groups: OVX control; ICA treatment; ICA $+5 \mathrm{mg} / \mathrm{l}$ ICI 182,780, a high-affinity ER antagonist, treatment; and Sham positive control. The osteogenic differentiation and mineralization of each group were determined by evaluation of ALP activity, calcium deposition, ALP staining and alizarin red staining of mineralized nodules at days 14 and 21 . All groups were cultured in an osteogenic medium contained $10^{-8} \mathrm{M}$ dexamethasone, $50 \mu \mathrm{g} / \mathrm{ml} \mathrm{L}-2$-ascorbic acid, and $10 \mathrm{~mm}$ $\beta$-glycerophosphate. The expression of the Runx-2 and OSX expression was also detected by western blotting and RT-qPCR, the cells were cultured for 7 days. The expression of factors involved in the estrogen signaling pathway, ER $\alpha$, progesterone receptor (PR) and trefoil factor 1 (PS-2), was also measured by western blotting and RT-qPCR. All materials and methods were identical to those described in the previous section.

ICA treatment the OVX-BMSC to recover the cell osteogenic differentiation and bone formation assay in vivo. The $0.8 \%$ collagen scaffolds used in the study were obtained from Rebone Biomaterial Co., Ltd. (Shanghai, China). Confluent BMSCs from OVX rats, Sham rats and OVX rats treated with ICA were detached from culture dishes, centrifuged to form cell pellets, and then resuspended in $0.8 \%$ collagen at a density of $2 \times 10^{7}$ cells $/ \mathrm{ml}$. Cell suspensions were transplanted into six four-week old male nude mice. Each mouse received the following three groups of complexes: Sham-BMSC group, OVX-BMSCs and ICA + OVX-BMSCs group. Four cases were included in each group. Mice were anesthetized by intramuscular injection of pentobarbital following inhalation of light ether. Longitudinal incisions were made on the back of each mouse and three separate subcutaneous pockets were subsequently created by blunt dissection. Finally, three complexes were randomly implanted into the pockets and the skins were sutured. At 12 weeks post-surgery, the implants were harvested, fixed in $10 \%$ buffered formaldehyde for $24 \mathrm{~h}$, decalcified in $10 \%$ EDTA, embedded in paraffin, sectioned into $4-\mu \mathrm{m}$ sections and stained with hematoxylin and eosin (H\&E). Photomicrographs of each section were captured with a light microscope (Olympus corporation, Tokyo, Japan).

Statistical analysis. The data are presented as the mean \pm standard deviation. Each treatment group had at least three samples $(n=3)$. All data were analyzed using one-way analysis of variance followed by the least significant difference post hoc test using SPSS version 16.0 (SPSS, Inc., Chicago, IL, USA). P<0.05 was considered to indicate a statistically significant difference.

\section{Results}

OVX-BMSC proliferation capacity is significantly decreased compared with Sham-BMSCs. At three months post-surgery, three rats from the OVX group and three from the Sham group were sacrificed. Micro-CT 3D reconstruction images showed that OVX rats had less trabecular bone, as well as disorganized trabecular architecture, expanded marrow cavities and thinning cortical bones compared with the bones of Sham rats (Fig. 1A). These results demonstrated that the creation of the osteoporotic animal model had been successful. BMSCs were isolated from Sham and OVX rats. Results of the colony-forming assay demonstrated that the Sham-BMSCs clone formation rate was $\sim 38.6 \%$, compared with the OVX-BMSCs clone formation rate of 22.8 . The Sham-BMSCs exhibited a stronger proliferation ability and began the proliferative process without a stagnation period. The logarithmic growth phase began at day 3, the growth peak was reached on day 7 and on day the growth plateau phase was entered (Fig. 1B).

OVX-BMSC osteogenic differentiation capacity is significantly decreased compared with Sham-BMSCs. In order to further evaluate osteogenic differentiation, the ALP activity and 
calcified tubercle red staining in the two groups was compared. The expression of osteogenic-associated genes and proteins was also detected in the Sham-BMSCs and OVX-BMSCs using RT-qPCR and western blotting analysis. The results showed that the Sham-BMSCs had significantly larger CFU-FALP than OVX-BMSCs (Fig 2A), The ALP activity was also higher in the Sham group than that in the OVX group (Fig 2B). Similarly, with ALP staining, the mineralized nodule formation assay demonstrated more and larger areas of mineralized nodules in the Sham-BMSCs group compared with the OVX-BMSCs group (Fig. 2C). The calcium deposition volume was also significantly higher in the Sham group than the OVX group (Fig. 2D). The expression of the osteogenic-associated genes, Runx-2 and OSX, was significantly higher in the Sham-BMSCs group than in the OVX-BMSCs group (Fig. 2E and F). The expression of the Runx-2 and OSX proteins was also stronger in the Sham-BMSCs group compared with the OVX-BMSCs group (Fig. 2G and H).

ICA treatment recovers the osteogenic differentiation of OVX-BMSCs through the estrogen pathway. The effects of ICA on the differentiation and mineralization of OVX-BMSCs were investigated by staining of mineralized bone nodules and ALP histochemical staining at days 14 and 21. ALP activity and mineralized bone nodules were used as a phenotypic marker for osteogenic differentiation, and were detected simultaneously. Following treatment of the OVX-BMSCs with 10P-5PM ICA for 14 days under the osteogenic inducing medium, the ICA treatment groups exhibited a significant increase in cellular ALP activity and in the area positive for ALP histochemical staining, compared with the OVX-BMSCs group. No significant difference was detected between the Sham-BMSCs and ICA treatment groups (Fig. 3A and B). However, when ICI was added to the ICA treatment group, the ALP activity and area positive for ALP histochemical staining was significantly lower than in the ICA treatment group alone. Furthermore, no significant difference was detected between the OVX-BMSCs and the ICI+ICA groups (Fig. 3A and B). The alizarin red staining of bone nodules and the volume of calcium sediment demonstrated that ICA stimulated the differentiation of OVX-BMSCs into mature mineralized osteoblastic cells. The same pattern was observed with the results of the ALP assay. No difference was detected between the Sham-BMSC and ICA treatment groups. However, these groups each exhibited more bone nodule and a greater volume of calcium sediment than the OVX-BMSCs group. In addition, these values were lower in the ICI+ICA group compared with the Sham-BMSC and ICA treatment groups (Fig. 3C and D).

Following culture with an osteogenic inducing medium for seven days, total mRNA was isolated from the BMSCs in each group. The expression of the PS-2, ER $\alpha$, PR, Runx-2 and OSX genes was detected by RT-qPCR. For PS-2, the relative levels of mRNA were consistently higher in the cultures grown in the presence of ICA compared with those in the OVX-BMSCs and ICI+ICA groups. also almost higher than Sham-BMSCs group (Fig. 3E). For $\mathrm{ER} \alpha$, the relative levels of $\mathrm{mRNA}$ were higher in cultures grown in the presence of ICA compared with those in the Sham-BMSCs and OVX-BMSCs groups. However, when ICI was added to the ICA treatment group, the ER $\alpha$ expression was significantly reduced compared with that in the other groups (Fig. 3F). For PR, the relative levels of mRNA were higher following ICA treatment of OVX-BMSCs, but this increase was abrogated by the administration of ICI. No significant difference was detected between the between the Sham and ICA treatment groups (Fig. 3G). For Runx-2 and OSX, as the lower reaches of the osteogenic differentiation marker gene was also detected, and they have the same expression pattern, the results showed that treatment with ICA significantly increased the expression of these genes compared with that of the OVX-BMSCs and ICI+ICA groups (Fig. 3H and I).

Secretion of the PS-2, ER $\alpha$, PR, Runx-2 and OSX proteins was assessed by western blot analysis of cultured BMSCs in each group. Under osteogenic-inducing conditions, the protein expression was detected as an immunoreactive blot and the area of blots was analyzed by IPP imaging software. The $\beta$-actin protein was analyzed in the same samples as an reference protein. Based on the area of the blots, the expression of these proteins in the OVX-BMSCs was compared with the Sham-BMSCs group. The expression of the estrogen pathway marker protein PS-2, $\mathrm{ER} \alpha$ and PR was reduced in the OVX-BMSCs group, in particular that of ER $\alpha$ expression. However, when the OVX-BMSCs were treated with ICA, the expression of PS-2, ER $\alpha$ and PR was restored. By contrast, when the ICA and ICI were administered simultaneously, the expression of $\mathrm{ER} \alpha$ was not significantly increased compared with the OVX-BMSCs group, and it was significantly lower than that in the Sham-BMSCs and ICA treatment group s (Fig. 4A-C). The expression of the Runx-2 and OSX proteins in the OVX-BMSCs group was also lower than that in the Sham-BMSCs group and ICA treatment group following culture in an osteogenic-inducing medium for seven days. Furthermore, the levels of these proteins decreased when ICI was added to the ICA treatment group (Fig. 4D and E).

ICA restores the ability for osteogenic differentiation and bone formation in BMSCs in vivo. At 12 weeks post-surgery, the implants were harvested, embedded in paraffin, sectioned into $4-\mu \mathrm{m}$ sections and stained with H\&E. The results demonstrated new bone formation in all three groups. However, there were marked differences among them. The OVX-BMSC group exhibited a smaller quantity of bone formation than the Sham-BMSC group. The LCA treatment group exhibited more bone formation than the OVX-BMSC group, and slightly less than the Sham-BMSC group (Fig. 5). Thus, the H\&E staining results showed that OVX-BMSC osteogenic differentiation and bone formation ability was also restored by treatment with ICA in vivo.

\section{Discussion}

ICA, a prenylated flavonol glycoside isolated from the Epimedium herb, stimulates osteogenic differentiation of BMSCs and inhibits the bone resorption activity of osteoclasts. The existence of a prenyl group on C-8 of the ICA molecule has been shown to lead to an increased potency of ICA with regard to osteogenic activity $(13,21,22)$. It has been reported that ICA induces osteogenic differentiation in a BMP-2-, SMAD 4-, Runx-2-, or ER-dependent manner $(23,27)$ and that it inhibits osteoclast differentiation and bone resorption via suppression of MAPKs/NF- $\kappa \mathrm{B}$ synthesis (15). In addition, ICA is a PDE5 inhibitor $(28,29)$ and is known to enhance the production of bioactive nitric oxide, as well as mimicking the effects of testosterone (26). 
A
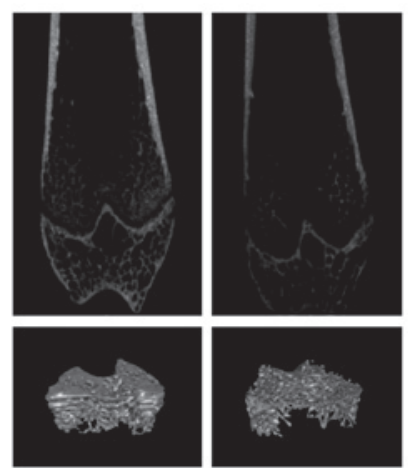

Sham
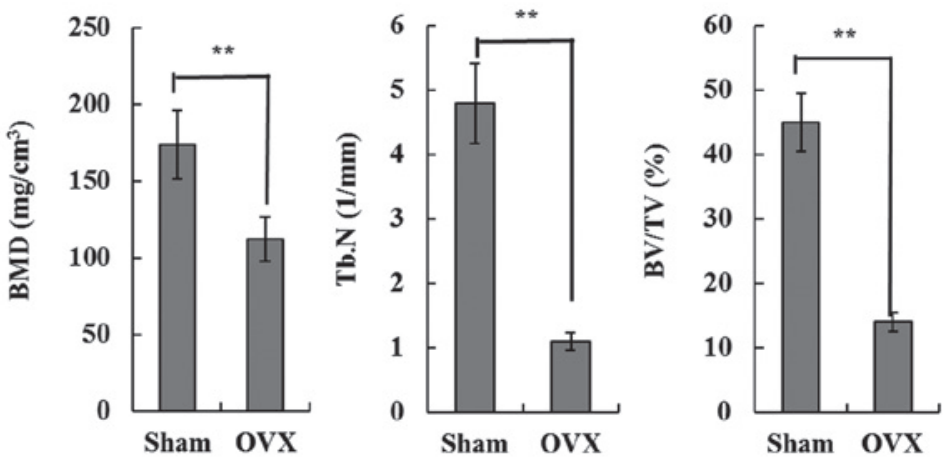

B
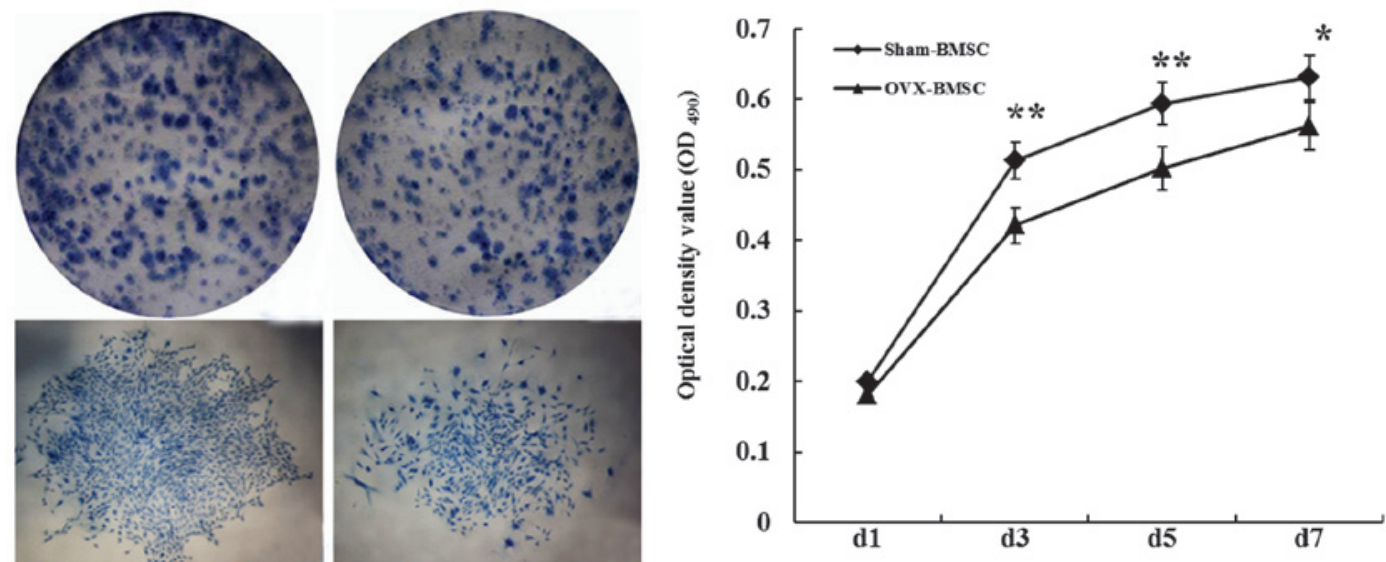

Figure 1. (A) OVX rats exhibited less trabecular bone, disorganized trabecular architecture, expanded marrow cavities and thinning cortical bones compared with the sham rats. (B) Results of the colony-forming experiments show higher clone formation and proliferation rate in the Sham-BMSCs group than in the OVX-BMSCs group formation. OVX, ovariectomy; BMSCs, bone marrow stromal cells.

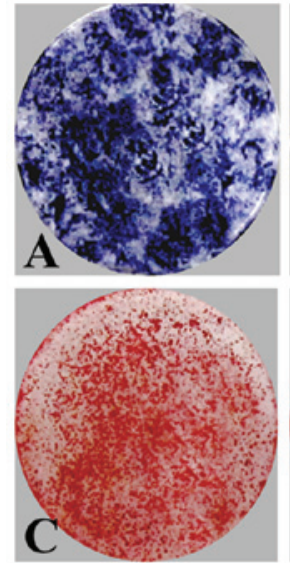

Sham-BMSC
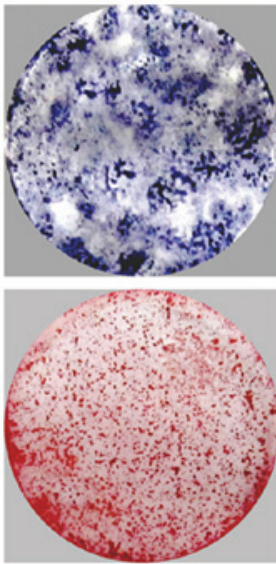

OVX-BMSC
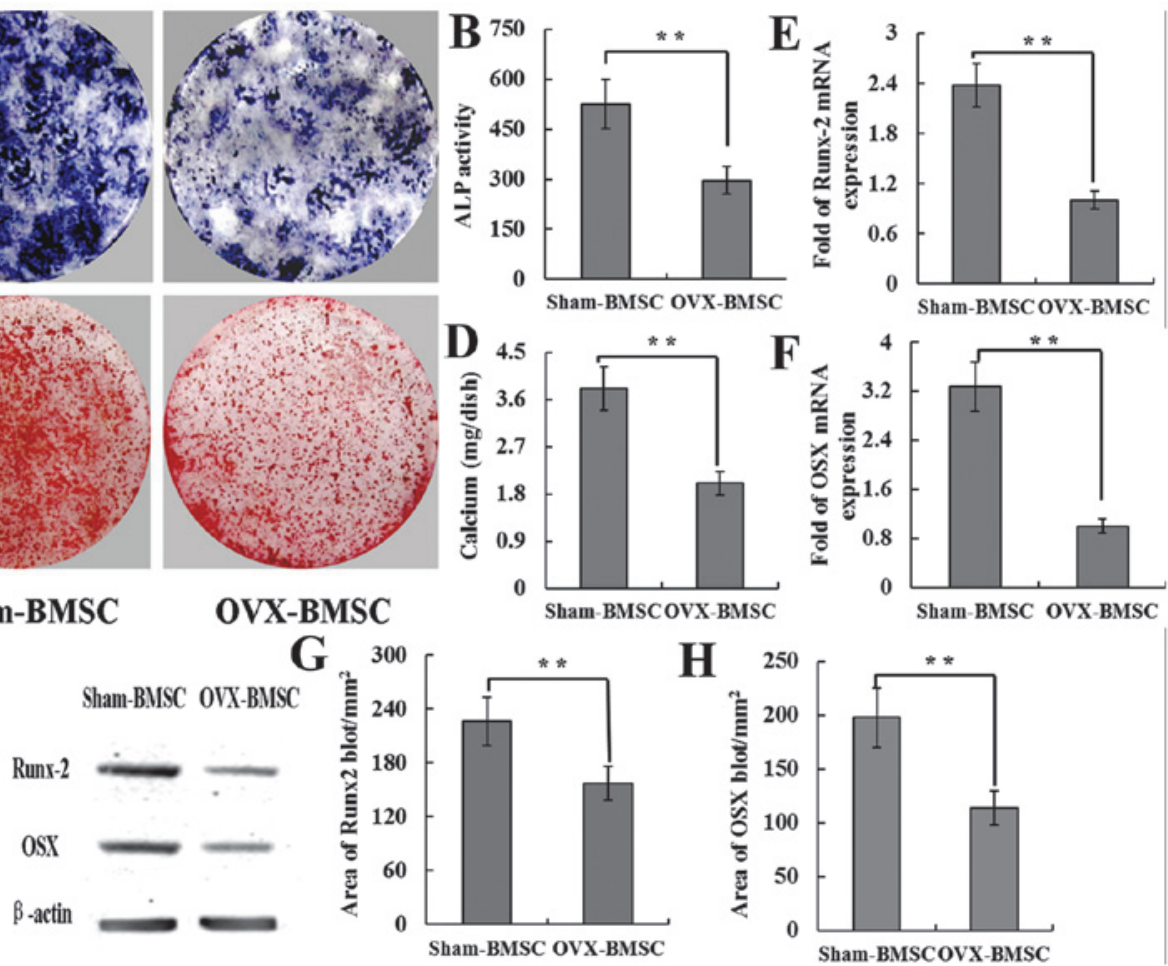

Figure 2. Sham-BMSCs exhibited a significantly larger area of (A) CFU-FALP colonies and (B) mineralized nodules than OVX-BMSCs. (C) ALP activity and (D) calcium deposition volume was significantly higher in the Sham-BMSCs group than in the OVX-BMSCs group. Expression levels of the osteogenic-associated genes, (E) Runx-2 and (F) OSX was significantly higher in the Sham-BMSCs group than those in the OVX-BMSCs group. The expression of the (G) Runx-2 and (H) OSX proteins exhibited the same pattern as that of the respective genes. CFU-FALP, number of colonies positive for ALP; ALP, alkaline phosphatase; BMSCs, bone marrow stromal cells; OVX, ovariectomy; OSX, osterix; Runx-2, runt-related transcription factor 2. 

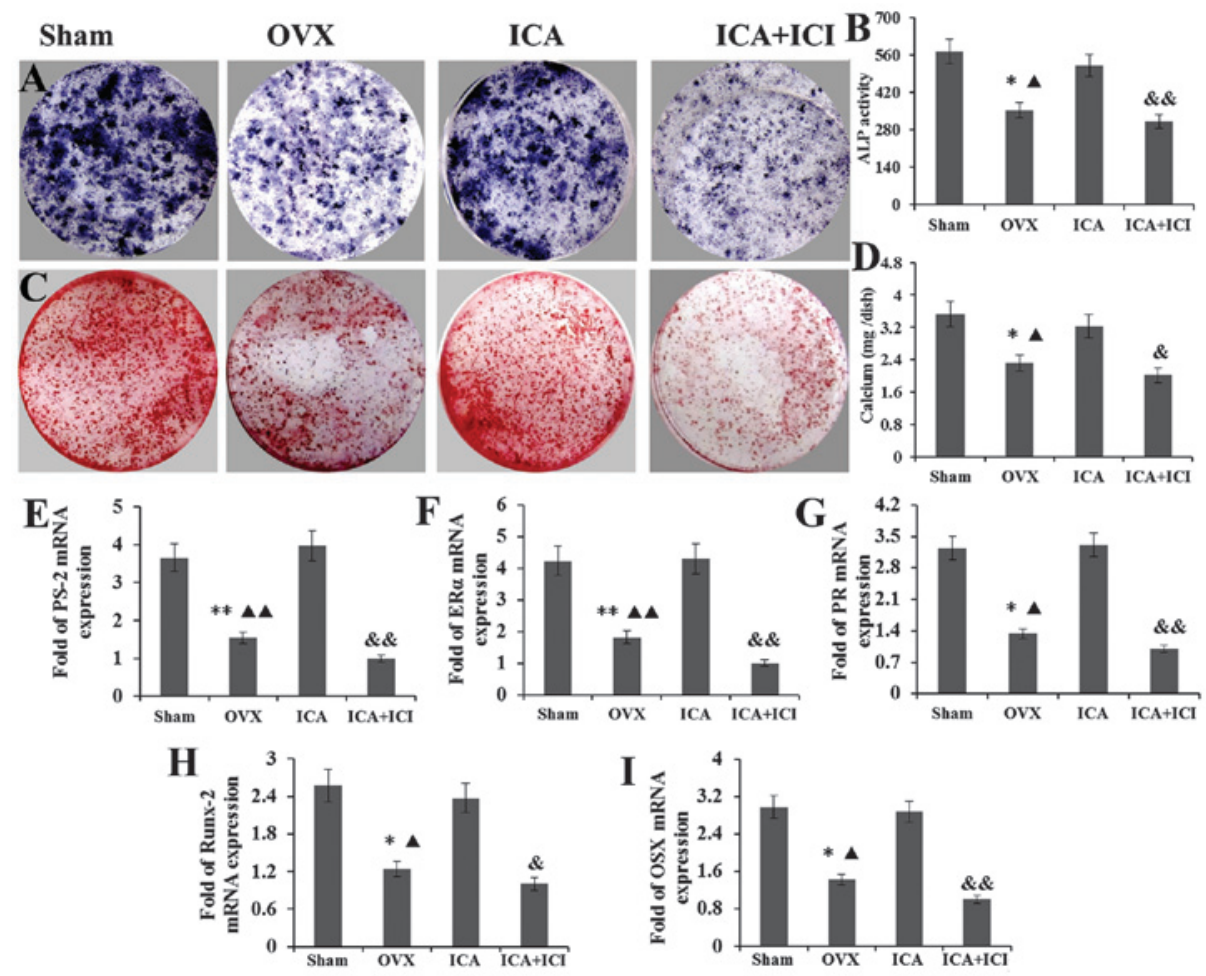

Figure 3. ICA treatment groups exhibited a large area positive for (A) ALP histochemical staining and (B) ALP activity compared with the OVX-BMSCs group No significant difference was detected between the Sham-BMSCs and ICA treatment groups. When ICI was added to the ICA treatment group, the ALP activity and area positive for ALP histochemical staining was significantly lower than that in the ICA treatment group, whilst no difference was detected between the OVX-BMSCs and ICI + ICA groups. (C) Alizarin red staining of bone nodule mineralization and (D) the volume of calcium sediment yield exhibited the same pattern as that of the ALP assay. No difference was detected between the Sham-BMSCs and ICA treatment groups, whilst those two groups exhibited more bone nodule mineralization and calcium volume than the OVX-BMSCs group. The results for the ICI + ICA group were lower than those for the Sham-BMSC and ICA treatment groups. The expression of the (E) PS-2, (F) ER $\alpha,(\mathrm{G}) \mathrm{PR},(\mathrm{H})$ Runx-2 and (I) OSX genes exhibited the same pattern. ICA, icariin; ALP, alkaline phosphatase; OVX, ovariectomy; BMSCs, bone marrow stromal cells; ICI, ICI 182,780; PS-2, trefoil factor 1; ER $\alpha$, estrogen receptor $\alpha$; PR, progesterone receptor; OSX, osterix; Runx-2, runt-related transcription factor 2.
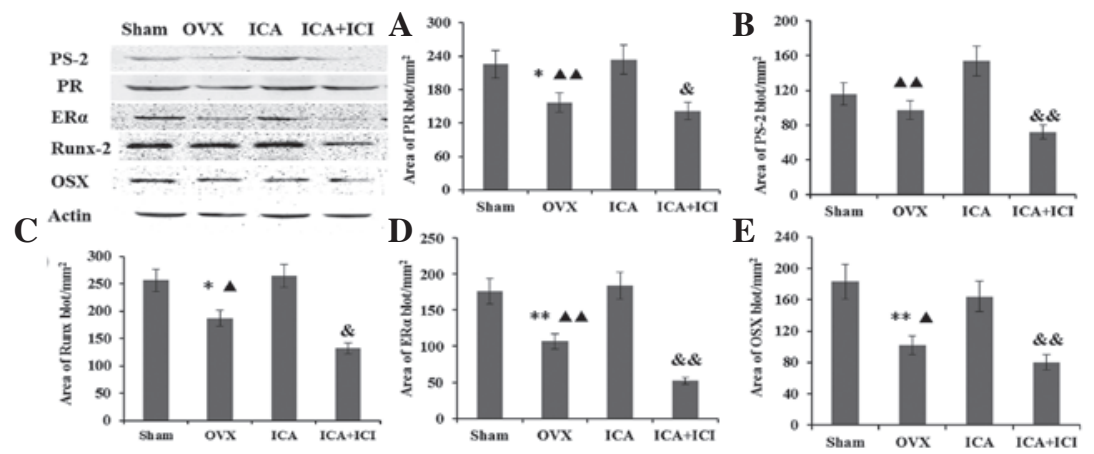

Figure 4. Expression of the (A) PR, (B) PS-2, (C) Runx-2, (D) ER $\alpha$ and (E) OSX proteins was assessed by western blot analysis of cultured BMSCs in the Sham, OVX, ICA treatment and ICI + ICA treatment groups. PS-2, trefoil factor 1; ER $\alpha$, estrogen receptor $\alpha$; PR, progesterone receptor; BMSCs, bone marrow stromal cells; OVX, ovariectomy; ICA, icariin; ICI, ICI 182,780; OSX, osterix; Runx-2, runt-related transcription factor 2.
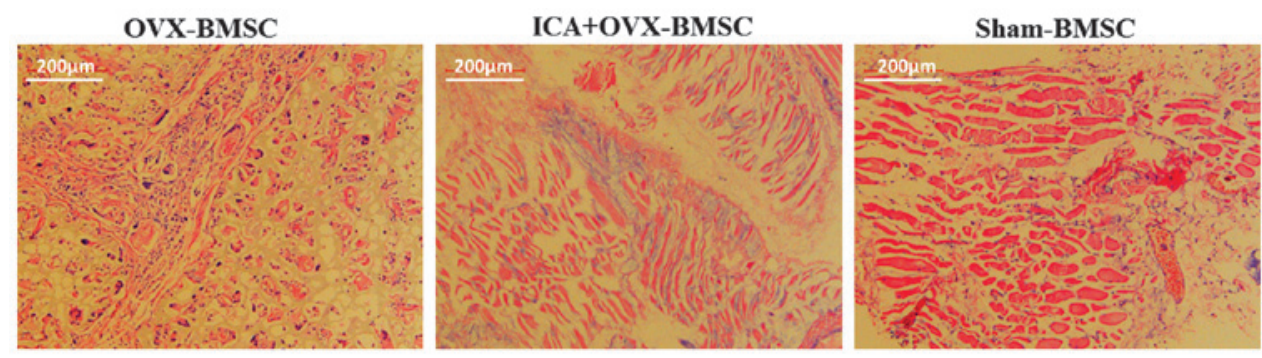

Figure 5. Sham-BMSC, OVX-BMSC and ICA treatment OVX-BMSC transplantation in nude mice, stained with hematoxylin and eosin. BMSC, bone marrow stromal cell; ICA, icariin; OVX, ovariectomy. 
In the present study, it was shown that cell proliferation, cell differentiation, mineralization capacity, and the expression of osteogenesis-related genes and proteins was significantly decreased in the OVX-BMSCs compared with the Sham-BMSCs. ICA was shown to improve the differentiation, mineralization, and expression of osteogenesis-related genes and protein in OVX-BMSCs. Furthermore, these effects were completely blocked by administration of the ER inhibitor, ICI 182780, demonstrating that the effects on proliferation of icaritin and desmethylicaritin were mediated by its action on the ER. Bian et al demonstrated that ICA may have different effects on BMSCs isolated from rats in which osteoporosis had been induced by corticosterone from that in those in which it had been induced by OVX. Although ICA treatment promoted osteogenic differentiation in BMSCs from corticosterone-treated and OVX rats, microarray studies of the isolated BMSCs showed that ICA treatment produced a marked shift in the expression of genes involved in cell communication, cell adhesion, the cell cycle and the secretion of cytokines, with this shift being more significant in the corticosterone-treated rats. Notably, there was little overlap between the differentially expressed genes induced by ICA treatment in these two models, suggesting that the effects, and molecular mechanisms underlying these effects, of ICA in reducing bone loss and prevention of osteoporosis may be pathogenesis-dependent (30). However, there is currently a lack of data on the influence on the ER of ICA, and the associated mechanisms. In the present study, the expression of ER $\alpha$, SP-2 and PR was detected following ICA treatment of OVX-BMSCs. The PS- 2 and PR genes are estrogen responsive in hepatocarcinoma cells (HepG2) in the presence of the ER (31-33). The result from the current study showed that the expression of the ER $\alpha, \mathrm{SP}-2$ and PR genes and proteins in OVX-BMSCs was upregulated by ICA treatment, and that the ER inhibitor, ICI 182780 , blocked the upregulation of these molecules. Therefore, it may be that ICA improves the osteogenic differentiation and mineralization of OVX-BMSCs in vitro via the ER pathway. Osteogenic differentiation and bone formation ability, which had been damaged by estrogen deficiency and increased age, was also restored in vivo.

\section{References}

1. McGarry KA and Kiel DP: Postmenopausal osteoporosis Strategies for preventing bone loss, avoiding fracture. Postgrad Med 108: 79-82, 85-88, 91, 2000.

2. Hertrampf T, Gruca MJ, Seibel J et al: The bone-protective effect of the phytoestrogen genistein is mediated via ER alpha-dependent mechanisms and strongly enhanced by physical activity. Bone 40: 1529-1535, 2007.

3. Negishi-Koga T, Shinohara M, Komatsu N, et al: Suppression of bone formation by osteoclastic expression of semaphorin 4D Nat Med 17: 1473-1481, 2011.

4. Uccelli A, Moretta L and Pistoia V: Mesenchymal stem cells in health and disease. Nat Rev Immunol 8: 726-736, 2008.

5. Liu Y, Wang L, Kikuiri T et al: Mesenchymal stem cell-based tissue regeneration is governed by recipient $\mathrm{T}$ lymphocytes via IFN- $\gamma$ and TNF- $\alpha$. Nat Med 17: 1594-1601, 2011

6. Rodan GA and Martin TJ: Therapeutic approaches to bone diseases. Science 289: 1508-1514, 2000.

7. Teitelbaum SL: Osteoclasts: what do they do and how do they do it? Am. J. Pathol 170: 427-435, 2007.

8. Riggs BL and Hartmann LC: Selective estrogen-receptor modulators - mechanisms of action and application to clinical practice. N Engl J Med 348: 618-629, 2003.

9. Couse JF and Korach KS: Estrogen receptor null mice: what have we learned and where will they lead us? Endocr Rev 20: 358-417, 1999.
10. Shang Y and Brown M: Molecular determinants for the tissue specificity of SERMs. Science 295: 2465-2468, 2002.

11. Mangelsdorf DJ, Thummel C, Beato M, et al: The nuclear receptor superfamily: the second decade. Cell 83: 835-839, 1995.

12. Belandia B and Parker MG: Nuclear receptors: a rendezvous for chromatin remodeling factors. Cell 114: 277-280, 2003.

13. Ming LG, Chen KM and Xian CJ: Functions and action mechanisms of flavonoids genistein and icariin in regulating bone remodelling. J Cell Physiol 228: 513-521, 2013.

14. Chen KM, Ge BF, Ma HP, et al: Icariin, a flavonoid from the herb Epimedium enhances the osteogenic differentiation of rat primary bone marrow stromal cells. Pharmazie 60: 939-942, 2005

15. Chen KM, Ma HP, Ge BF, et al: Icariin inhibits the osteoclast formation induced by RANKL and macrophage-colony stimulating factor in mouse bone marrow culture. Pharmazie 62: 388-391, 2007.

16. Yamaza T, Miura Y, Bi Y, et al: Pharmacologic stem cell based intervention as a new approach to osteoporosis treatment in rodents. PLoS One 3: e2615, 2008.

17. Hsieh TP, Sheu SY, Sun JS and Chen MH: Icariin inhibits osteoclast differentiation and bone resorption by suppression of MAPKs/NF- $\kappa$ B regulated HIF- $1 \alpha$ and PGE(2) synthesis. Phytomedicine 18: 176-185, 2011.

18. Huang J, Yuan L, Wang X, et al: Icaritin and its glycosides enhance osteoblastic, but suppress osteoclastic, differentiation and activity in vitro. Life Sci 81: 832-840, 2007.

19. Zhai YK, Ge BF and Ma HP: Comparative study on the osteogenic differentiation of rat bone marrow stromal cells effected by icariin and icariside II. Zhong Yao Cai 33: 1896-1900, 2010 (In Chinese).

20. Zhai YK, Ge BF and Ma HP: Icariin promotes osteogenic differentiation of rat bone marrow stromal cells in vitro. Zhongguo Zhong Yao Za Zhi 35: 3219-3222, 2010 (In Chinese).

21. Ma HP, Ming LG and Ge BF: Icariin is more potent than genistein in promoting osteoblast differentiation and mineralization in vitro. J Cell Biochem 112: 916-923, 2011.

22. Ming LG, Zhou J, Cheng GZ, et al: Osthol, a coumarin isolated from common cnidium fruit, enhances the differentiation and maturation of osteoblasts in vitro. Pharmacology 88: 33-43, 2011.

23. Mok SK, Chen WF and Lai WP: Icariin protects against bone loss induced by oestrogen deficiency and activates oestrogen receptor-dependent osteoblastic functions in UMR 106 cells, Br J Pharmacol 159: 939-949, 2010.

24. Powell ME and Smith MJ: The determination of serum acid and alkaline phosphatase activity with 4-aminoantipyrine (A.A.P.). J Clin Pathol 7: 245-248, 1954

25. Nian H, Ma MH, Nian SS and Xu LL: Antiosteoporotic activity of icariin in ovariectomized rats. Phytomedicine 16: 320-326, 2009.

26. Zhang ZB and Yang QT: The testosterone mimetic properties of icariin. Asian J Androl 8: 601-605, 2006.

27. Ning H, Xin ZC, Lin G, et al: Effects of icariin on phosphodiesterase-5 activity in vitro and cyclic guanosine monophosphate level in cavernous smooth muscle cells. Urology 68: 1350-1354, 2006.

28. Dell'Agli M, Galli GV, Dal Cero E, et al: Potent inhibition of human phosphodiesterase-5 by icariin derivatives. J Nat Prod 71: 1513-1517, 2008.

29. Xu HB and Huang ZQ: Icariin enhances endothelial nitric-oxide synthase expression on human endothelial cells in vitro. Vascul Pharmacol 47: 18-24, 2007.

30. Bian Q, Huang JH, Liu SF, et al: Different molecular targets of icariin on bMSCs in CORT and OVX-rats. Front Biosci (Elite Ed) 4: 1224-1236, 2012.

31. Barkhem T, Haldosén LA, Gustafsson JA and Nilsson S: Transcriptional synergism on the pS2 gene promoter between a p160 coactivator and estrogen receptor-alpha depends on the coactivator subtype, the type of estrogen response element, and the promoter context. Mol Endocrinol 16: 2571-2581, 2002.

32. Zampese E, Fasolato C, Pozzan T and Pizzo P: Presenilin-2 modulation of ER-mitochondria interactions: FAD mutations, mechanisms and pathological consequences. Commun Integr Biol 4: 357-360, 2011.

33. Hsieh TP, Sheu SY, Sun JS, et al: Icariin isolated from Epimedium pubescens regulates osteoblasts anabolism through BMP-2, SMAD4, and Cbfa1 expression. Phytomedicine 17: 414-423, 2011 